\title{
ANÁLISE DO IMPACTO DE UM PROGRAMA DE EDUCAÇÃO EM SAUDE SOBRE O PERFIL LIPIDICO DE IDOSOS
}

\section{IMPACT ANALYSIS OF A HEALTH EDUCATION PROGRAM ON THE LIPID PROFILE OF ELDERLIES}

Fabiana Postiglione Mansani* Hendrick Luiz Scharneski**

\begin{abstract}
Resumo: A tendência ao envelhecimento da população brasileira leva a focar nos distúrbios cardiovasculares, que são muito prevalentes entre idosos. As dislipidemias estão entre seus fatores de risco e podem ser controladas com hábitos de vida saudáveis. Objetivou-se verificar as alterações no perfil lipídico de idosos após atividades relacionadas com o incentivo ao estilo de vida saudável. Para isso, foram realizadas 7 atividades junto a um grupo de idosos participantes do projeto de extensão denominado "São Vicente: para uma melhor qualidade de vida", visando estimular hábitos de vida saudáveis e, assim, melhorar os parâmetros lipídicos. Foram levantados os valores lipídicos dos participantes e comparados com os valores encontrados após essas intervenções. Encontrou-se queda da lipoproteína de alta densidade e triglicerídeos. Conclui-se que a proposta de mudança dos hábitos de vida junto à população idosa pode resultar em alterações importantes sobre algumas classes de lipoproteínas.
\end{abstract}

Palavras chave: Envelhecimento; Lipídeos; Estilo de Vida.

\begin{abstract}
The Brazilian population aging trend has led the health sector to focus on cardiovascular disorders, which are highly prevalent among elderlies. Dyslipidemias are among their factor risks and can be controlled with a healthy lifestyle. This study aimed at verifying changes in a group of elderlies' lipid profile after carrying out activities incentivizing a healthy lifestyle. To reach the objectives, 7 activities were developed with an elderly group that take part in an extension project called 'São Vicente: for a better quality of life' (São Vicente: Para uma melhor qualidade de vida), with the purpose of stimulating healthy life habits and, thus, improve their lipid parameters. Before the interventions, the lipid values of the participants were investigated and compared with the values found after the activities had been completed. The results showed decrease in the values of high density lipoproteins and triglycerides, which led to the conclusion that the proposal of changes in the elderlies' lifestyle can result in important changes in some lipoprotein classes.
\end{abstract}

Keywords: Aging; Lipids; Lifestyle. 


\section{Introdução}

A população senil está crescendo no Brasil, o que aponta uma melhoria da qualidade de vida do brasileiro. Com isso, associada a uma redução das taxas de natalidade, há uma alteração no perfil etário populacional, que vai deixando de ser predominantemente jovem para dar lugar a uma proporção aumentada de idosos (VERAS, 2012, p.1834-1840). De acordo com estimativas do Instituto Brasileiro de Geografia e Estatística - IBGE (2010), a população de idosos deve chegar a cerca de 65 milhões de pessoas ou $29,7 \%$ da população brasileira no ano de 2050. Segundo Mendes, Walksman e Farah (2014, p.862), o grupo que mais ganha espaço nas estatísticas demográficas é o de idosos muito velhos (80 anos ou mais), formado em sua maioria por mulheres, processo denominado de feminilização da velhice.

Concomitante a essa mudança no perfil etário populacional, há uma alteração do quadro mórbido, que deixa de ser representado pelas doenças infectocontagiosas e passa a ser representado pelas doenças crônico-degenerativas.

Entre estas doenças crônicas figuram os distúrbios cardiovasculares, que são responsáveis por até $35 \%$ da mortalidade em idosos com 80 anos ou mais e em torno de $32 \%$ entre idosos entre 60 e 69 anos (OLIVEIRA; MEDEIROS; LIMA, 2015, p.8594). Isso porque, além das alterações circulatórias decorrentes do próprio envelhecimento, a senilidade está associada a uma grande prevalência de fatores de risco, entre eles as dislipidemias (KRAUSE et al., 2008, p. 327-332; FERREIRA et al., 2010, p. 621$628)$.

Além disso, estudos nacionais e internacionais mostraram que grande parte dos indivíduos que fazem uso de medicação hipolipemiante de maneira isolada não conseguiu atingir suas metas lipídicas. Isso demonstra a necessidade de associar o tratamento medicamentoso à mudança dos hábitos de vida, a qual inclui dieta e exercícios físicos (VACANTI et al., 2005, p. 162-5; NAGASHIMA; KAZANUKI, 2005; p. 338-342; YUSUF et al., 2004, p. 937-52, GRUNDY et al., 2004, p.227-39).

Vários estudos têm comprovado que programas de incentivo à mudança nos hábitos de vida têm impacto positivo sobre a redução de admissões hospitalares, qualidade de vida, capacidade funcional e prevenção de infarto em indivíduos com fatores de risco coronarianos, reduzindo consideravelmente os gastos públicos (ROMERO, 2000, p.923-934; MCLISTER et al., 2001; p.957-62.; PIRICH; BISOVSKY; SINGINGER, 1992, p.401-8;).

Porém, essa modalidade de atenção ainda é subutilizada devido à falta de conhecimento de sua eficácia, que deve ser comprovada e divulgada por meio de pesquisas como a que foi desenvolvida neste trabalho, que utiliza uma modalidade inovadora de incentivo a mudanças nos hábitos de vida.

\section{Objetivo}

O objetivo desta pesquisa é identificar as alterações no perfil lipídico decorrentes de um programa de atividades de educação em saúde junto a um grupo de idosos.

\section{Métodos}

\section{Estudo e seleção da amostra}

Realizou-se um estudo intervencionista. Após aprovação do comitê de ética em pesquisa da Universidade Estadual de Ponta Grossa, sob o parecer número 1.085.339, os idosos de um grupo de convivência do munícipio de Ponta Grossa, Paraná, foram convidados a participar da pesquisa.

Foram incluídos idosos dislipidêmicos e não dislipidêmicos, que por meio da assinatura do termo de consentimento livre e esclarecido aceitaram a análise de seu perfil lipídico e a participação nas atividades. Foram excluídos os idosos que faltaram em uma das coletas sanguíneas. A classificação em dislipidêmicos ou não se baseou na $5^{\text {a }}$ Diretriz Brasileira de Dislipidemias e Prevenção da Aterosclerose da Sociedade Brasileira de Cardiologia.

\section{Levantamento de dados}

Inicialmente, por meio de questionários, foram levantados dados gerais da população, como idade, renda, sexo, índice de massa corpórea, comorbidades, história mórbida, história mórbida familiar. Foram realizadas coletas sanguíneas para aferição do CT (Colesterol total), HDL (High Density Lipoprotein), LDL (Low Density Lipoprein) e TG (triglicerídeos) dos participantes em duas ocasiões: uma anteriormente ao início das intervenções e outra posteriormente.

Assim, o intervalo entre uma aferição de perfil lipídico e outra foi de 5 meses. Em cada uma delas, os participantes foram orientados a se dirigir à sede de encontros do grupo, onde ocorreram as coletas, após 12 horas em jejum. As duas análises seguiram a mesma metodologia e foram realizadas no laboratório do Hospital Universitário Regional dos Campos Gerais, utilizando-se o equipamento CT600 Wiener e metodologia enzimática colorimétrica para $\mathrm{CT}, \mathrm{TG}$ e HDL colesterol, já o cálculo para LDL foi através da fórmula de Friedwald: LDL-c $=\mathrm{CT}(\mathrm{HDL}-\mathrm{c}+\mathrm{TG} / 5)$.

\section{Intervenções}

Foram feitas atividades que visaram à educação popular em saúde, distribuídas em um programa de 7 encontros, com intervalos não regulares entre um encontro e outro, que envolveram a prática de 3 caminhadas, 1 palestra, 1 bingo e 2 cozinhas lúdico- 
interativas. Essas últimas envolveram chá verde, e outra, suco verde.

A primeira caminhada durou 14 minutos, a segunda 12 e a terceira 11 . O percurso de 250 metros foi o mesmo nas três ocasiões, sempre no plano. Após cada caminhada, foi feito alongamento dos membros superiores e inferiores. Entre os pacientes diabéticos, tomou-se o devido cuidado com hidratação adequada durante a prática e, aqueles que tiveram sensibilidade alterada nos pés detectada com exame físico, foram aconselhados a usar calçados confortáveis durantes as caminhadas.

A palestra durou cerca de uma hora, quando os idosos receberam informações sobre as dislipidemias, suas possíveis complicações e os benefícios de mudanças de hábitos de vida. Foram estimulados a cessar o tabagismo, a ingestão excessiva de bebidas alcóolicas, a comer moderadamente, diminuindo a ingestão de carne vermelha, gordura animal, bolos, queijos amarelos, manteigas, sorvetes, e passarem a utilizar alimentos como farinha de trigo integral, peixe, legumes e verduras (KRIS-ETHERTON; HARRIS; APPEL, 2002, p.106-2747; BURR; BUTLAND, 1988, p.830-2; WEST; HAYES, 1968, p.853-62; DE BIASE et al., 2007, p.35-9; CHEN et al., 2008, p.138-44). Também se incentivou a prática regular de exercícios físicos, com uma frequência mínima de 4 vezes por semana durante 30 minutos, aumentando a intensidade dos exercícios gradativamente (SCHULER; ADAMS; GOTO, 2013, p.634-637).

Nas cozinhas interativas, os idosos foram orientados a preparar suco e chá verde, pelo impacto positivo que os mesmos têm sobre o perfil lipídico e a gordura corporal (JÚNIOR; LEMOS, 2010, p.174-178; BATISTA et al., 2009, p.128-134). O suco verde continha couve, gengibre, suco de limão e hortelã. $O$ chá verde foi preparado com ramos de hortelã fresca, suco de limão, chá verde e suco de maçã.

$\mathrm{O}$ bingo consistiu em 23 perguntas gerais sobre dislipidemias, dando-se mais ênfase aos cuidados de prevenção das dislipidemias. As perguntas foram numeradas e ordenadas por sorteio e, após cada uma, os idosos foram estimulados a responder e a procurar a imagem correspondente em suas cartelas, com o intuito de tornar o jogo educativo. Venceu quem completou a cartela.

As intervenções foram realizadas de outubro de 2015 a março de 2016.

\section{Projeto São Vicente: para uma melhor qualidade de vida}

O Projeto São Vicente surgiu inicialmente como um projeto extensionista, desenvolvido por acadêmicos dos cursos de medicina e enfermagem, sob a orientação de uma professora lotada no Departamento de Medicina da Universidade Estadual de Ponta Grossa. O Projeto realizava atividades de acompanhamento e observação junto a um grupo de idosos, previamente organizado com encontros semanais em uma sede própria, para ação social de integração e humanização.

O primeiro ano de desenvolvimento do Projeto despertou o interesse dos agentes, acadêmicos e professora a realizarem intervenções para verificar a possibilidade de melhoria da qualidade de vida dos idosos. Nesta etapa, foram formuladas as alterações na metodologia do Projeto, em que onde foram propostas atividades de intervenção junto ao grupo de idosos, e passou-se a mensurar de forma analítica as atividades desenvolvidas com cunho extensionista. Surgiu, então, a interface entre ensino, extensão e pesquisa.

Esta nova fase foi desenvolvida por uma equipe multiprofissional, além dos acadêmicos dos cursos de medicina e enfermagem que já integravam o projeto de extensão. Os encontros ocorreram aos sábados à tarde, junto a sede de encontro dos idosos, que pertence à Sociedade Vila Vicentina. Além do enfoque sobre doenças cardiovasculares, o grupo trabalhou também com outras morbidades bastante prevalentes entre idosos, como osteoporose e fraturas, obesidade, diabetes mellitus, tonturas e distúrbios cognitivos. Diante da possibilidade de levantar dados e da possível alteração dos parâmetros pelas atividades realizadas evidenciadas por dados da literatura, surgiu a ideia de realizar uma pesquisa envolvendo essa eventual alteração na população participante e, com seus resultados, analisar a necessidade de reformular as atividades a fim de proporcionar melhor benefício à sua saúde.

\section{Análise estatística}

Os dados qualitativos foram apresentados em número absoluto e proporções. Os dados quantitativos foram testados para a normalidade e presença de valores espúrios. Os dados foram relatados em média e desvio-padrão. Para comparação entre os valores laboratoriais, foi utilizado o teste $\mathrm{T}$ de student pareado. Para fins de cálculos, foi utilizado o programa MedCalc 5.1 (Bélgica).

\section{Resultados e discussão}

\section{Participantes}

Dos 22 idosos que iniciaram o estudo, 15 fizeram as duas coletas de sangue e tiveram seus resultados incluídos na análise estatística; nestes, a idade variou entre 68 e 84 anos, com uma média de 73,7 anos e desvio padrão de 4,20. Com relação ao sexo, 13 são do sexo feminino e 2 do sexo masculino. Quanto à renda, $80 \%$ da amostra $(\mathrm{n}=12)$ foram de um ou dois salários mínimos, $6,6 \%(\mathrm{n}=1)$ maior que dois 
salários mínimos e $13 \%(\mathrm{n}=2)$ não informaram a renda. Ainda, 12 foram considerados dislipidêmicos, e destes, 11 devido ao HDL diminuído, isoladamente ou em associação com LDL e/ou TG aumentados; e 3 idosos eram normolipidêmicos.

\section{Atividades}

A primeira sessão de caminhada teve boa aderência, com 12 participantes. Já a segunda e a terceira não tiveram boa aceitação por parte dos idosos, devido possivelmente à falta de motivação para realização das mesmas. Os dados com a adesão às intervenções estão registrados na Tabela 1.

Tabela 1 - Adesão dos participantes nas atividades desenvolvidas durante as intervenções

\begin{tabular}{|l|c|}
\hline \multicolumn{1}{|c|}{ Modalidade de Atividade } & Participação dos ldosos (\%) \\
\hline Cozinha lúdica (chá verde) & 87 \\
\hline Palestra & 87 \\
\hline Primeira caminhada & 80 \\
\hline Bingo & 80 \\
\hline Cozinha lúdica (suco verde) & 66 \\
\hline Segunda caminhada & 20 \\
\hline Terceira caminhada & 33 \\
\hline
\end{tabular}

\section{Impacto no perfil lipídico}

Não foram encontradas alterações estatisticamente significativas em nenhum dos componentes pesquisados, exceto o HDL colesterol, que diminuiu de maneira estatisticamente significativa, e os triglicerídeos, que também tiveram redução importante. Os valores encontrados estão mostrados na Tabela 2:

Tabela 2 - Efeitos das intervenções sobre as médias dos valores dos subtipos de lipoproteínas plasmáticas

\begin{tabular}{|l|l|l|l|}
\hline $\begin{array}{c}\text { Lipoproteína } \\
(\mathrm{mg} / \mathrm{dl})\end{array}$ & $\begin{array}{c}\text { Pré-intervenção } \\
\text { (média } \pm \mathrm{DP})\end{array}$ & $\begin{array}{c}\text { Pós-intervenção } \\
\text { (média } \pm \mathrm{DP})\end{array}$ & $p$ \\
\hline $\mathrm{CT}$ & $199,06 \pm 33,54$ & $186,4 \pm 31$ & $p=0,21$ \\
\hline $\mathrm{LDL}$ & $117 \pm 24,98$ & $118,93 \pm 26,53$ & $p=0,79$ \\
\hline HDL & $44,5 \pm 8,32$ & $41,73 \pm 8,7$ & $p=0,0095$ \\
\hline TG & $167,2 \pm 79,44$ & $148,86 \pm 59,96$ & $p=0,32$ \\
\hline
\end{tabular}

CT - colesterol total; LDL (low density lipoprotein) - colesterol de baixa densidade HDL (high density lipoprotein) - colesterol de alta densidade e TG - triglicerídeos.

A amostra foi composta por uma população idosa, a maioria do sexo feminino, de baixa renda e dislipidêmicos, sendo que a maioria foi devido ao HDL baixo.

A baixa renda é apontada por Oliveira e Pedroso (2014) como um fator de risco para distúrbios cardiovasculares, entre eles as dislipidemias, haja vista que esses distúrbios são mais prevalentes em populações menos privilegiadas socioeconomicamente.

Duarte (2007) mostrou que as dislipidemias e outros distúrbios cardiovasculares são mais prevalentes e respondem pela maior causa de morbimortalidade em mulheres acima de 50 anos devido, possivelmente, a alterações metabólicas decorrentes da menopausa.

Esses dados revelaram que a amostra deste trabalho foi bastante representativa da população pesquisada.

Quanto à aderência dos idosos às atividades da pesquisa, segundo a Tabela 1 , verificou-se grande aceitação dos idosos à palestra e à cozinha lúdica envolvendo o chá verde. Também se constatou que a participação dos idosos nas sessões de caminhada decaiu significativamente entre a primeira e segunda sessão, com $80 \%$ de participação na primeira sessão e $20 \%$ na segunda. Já na terceira sessão, houve um pequeno aumento de participação, com 33\% dos idosos. As atividades que não demandavam esforço físico, como palestra, chá verde, caminhada, bingo e suco verde obtiveram sempre boa aceitação. Houve evasão entre uma avaliação de perfil lipídico e outra, sendo que 22 indivíduos fizeram a primeira coleta de sangue para análise e apenas 15 fizeram a segunda, com perda de 7 indivíduos da amostra.

Segundo Chaves et al. (2015), que também fizeram intervenções educativas relacionadas com alterações nos hábitos de vida, a perda de amostra foi ainda mais significativa. $\mathrm{O}$ estudo iniciou com 15.073 participantes e encerrou com apenas $460(97 \%$ de desistência).

$\mathrm{O}$ que mais os participantes relataram como motivo de não adesão foi a limitação física, devido a dores e doenças e a indisposição devido à prática de exercícios no dia. Já em um estudo realizado por Krug et al. (2015), foram levantadas possíveis barreiras à prática de caminhada. São elas: a limitação física, a falta de disposição, o excesso de cuidado da família, as doenças, a falta de segurança, o casamento e o cuidar dos filhos, nunca ter realizado atividades físicas para o lazer, o medo de quedas e a idade. Além disso, a amostra do presente estudo foi composta, em sua maioria, por mulheres e, de acordo com o IBGE (c), elas têm menor aderência a atividades físicas do que o público masculino.

Com relação ao impacto das intervenções sobre os valores lipídicos, houve diminuição significativa no colesterol HDL, TG e nenhuma alteração significativa no CT e LDL colesterol (Tabela 2).

O valor médio dos TG diminuiu de 167,2 para 148,86. Essa redução é um achado importante, pois, em altos níveis, essas moléculas são um importante fator de risco coronariano. Isso porque determinadas partículas ricas em TG têm potencial aterogênico, principalmente em associação com HDL baixo, o que foi identificado aqui. Inúmeros estudos têm colocado os TG como preditor independente do risco coronariano (CHAVES et al., 2015).

Verificou-se redução nos valores de HDLcolesterol, de uma média de 44,5 antes das intervenções para 41,73 após as intervenções. Foi 
também um dado relevante, haja vista que o HDL desempenha importante ação antiaterogênica, inibindo o início e a progressão da doença arterial coronariana. Isso ocorre em virtude de sua capacidade de transporte reverso do colesterol aterogênico para o fígado, suas propriedades antioxidantes que impedem a oxidação do LDL que dá origem à formação do ateroma, sua ação anti-inflamtória sobre a viscosidade sanguínea e sobre o endotélio e redução da síntese de tromboxanos e prostaglandinas (KRUG; LOPES; MAZO, 2015; CONROY et al., 2003; MACKNESS; MACKNESS; DURRINGTON, 2000). Um outro estudo mostrou que o HDL baixo por um período de oito anos foi fator de risco significativo para desfechos cardiovasculares (RADER, 2003).

No estudo de Scherr et al. (2009) feito com pacientes com diagnóstico de doença arterial coronariana de um hospital especializado em distúrbios cardíacos, diferentemente do presente estudo, encontrou-se queda significativa de todos os componentes lipoproteicos dos participantes após palestras sobre fatores de risco coronarianos. Porém, assim como no presente trabalho, o colesterol HDL caiu significativamente $(\mathrm{p}=0,002)$.

O estudo de Ornish et al. (1990) obteve resultados positivos no colesterol total (queda de $24,3 \% ; \mathrm{p}=0,0192$ ) e LDL colesterol (queda de 37,4\%; $\mathrm{p}=0,0072)$ sobre o perfil lipídico somente com intervenções educativas (1990). Já Rutledge et al. (1999), através de intervenções no estilo de vida de 43 homens e mulheres coronariopatas, demonstraram redução do colesterol total e do LDL colesterol em comparação com o grupo controle composto por 28 indivíduos. O presente estudo se assemelhou ao de Rutledge et al. (1999) no sentido de que a amostra continuou em seu ambiente natural, sem confinação em um hotel, como no estudo de Ornish et al. Sendo assim, os pacientes foram analisados em seu ambiente natural.

Damião et al. (2011) analisaram atividades relacionadas com o incentivo à mudança para uma alimentação saudável, durante dois anos, com nipobrasileiros com e sem síndrome metabólica. Houve redução significativa no CT e LDL colesterol, porém, assim como no presente estudo, houve também redução no HDL colesterol.

Fett et al. (2009) realizaram um trabalho com mulheres sedentárias com idade média de 36 anos e com maior frequência, duração e alternância de exercícios entre aeróbios e resistidos. Verificou-se redução do $\mathrm{CT}$ e, assim como no presente trabalho, houve também redução da proteína da alta densidade e não se notou alteração no LDL e dos TG.

No presente trabalho, utilizaram-se ações inovadoras junto à comunidade de idosos. Porém, evidenciou-se que uma estratégia de educação em saúde pode falhar no sentido de não influenciar positivamente na saúde do indivíduo. Em estudo de Toledo et al. (2013), que avaliou a adesão à incorporação de um estilo de vida saudável proveniente de atividades que incentivaram, resultou em uma adesão de 50,9\%, incluído adesão parcial ou total. Mas quando se considera somente adesão total, essa porcentagem cai para apenas $17,6 \%$. Os que não seguiram orientação alguma, seja porque tentaram seguir e não conseguiram ou por sequer terem tentado, consistiram em $48,3 \%$ dos participantes.

Esses dados fortalecem a tese de que os programas de educação em saúde podem não conseguir incentivo à incorporação dos hábitos de vida saudável e, assim, falhar nos seus objetivos de redução do risco de doenças, o que provavelmente ocorreu neste estudo.

Os resultados aqui mostrados foram limitados pela amostra reduzida.

\section{Conclusão}

Concluiu-se que as atividades de educação em saúde relacionadas à incorporação de hábitos de vida saudáveis podem alterar significativamente alguns componentes lipídicos. Não houve alteração em todos os parâmetros, devido, provavelmente, a não incorporação no cotidiano dos participantes dos ensinamentos passados. Isso foi evidenciado pelo baixo interesse dos idosos em participar de atividades como a caminhada. Diante disso, analisou-se a possibilidade de ampliar as variedades de modalidades de atividades físicas, inserindo-se natação, ginástica e danças e a inclusão de um ou mais acadêmicos de educação física no Projeto, a fim de orientar a realização das mesmas. Essa readequação objetiva abranger os interesses e possibilidades de todos os idosos envolvidos, torná-los mais participativos e, assim, despertar maior interesse em incorporar a prática esportiva realizada a seus cotidianos.

\section{Referências}

BATISTA, G.A.P. et al. Estudo prospectivo, duplo cego e cruzado da camellia sinensis (chá verde) nas dislipidemias. Arquivos Brasileiros de Cardiologia, v. 93, n. 2, p. 128$134,2009$.

BURR, M.L.; BUTLAND, B.K. Heart disease in British vegetarians. The American Jornal of Clinical Nutricion, v.48, n.3, p.830-2, 1988.

CHAVES, G. et al. Educação para um estilo de vida saudável melhora sintomas e fatores de risco cardiovasculares - Estudo AsuRiesgo. Arquivos Brasileiros de Cardiologia, v.104, n.5, p.347-355, 2015.

CHEN, C.W. et al. Total cardiovascular risk profile of Taiwanese vegetarians. European Journal of Clinical Nutrition, v.62, n.1, p.138-44, 2008.

CONROY, R.M. et al. Estimulation of ten-year risk of fatal cardiovascular disease in Europe: the SCORE Project.

European Heart Journal, v.24, n.11, p.987-1003. 2003. 
DAMIÃO, R. et al. Impacto de um programa de intervenção sobre o estilo de vida nos perfis metabólico, antropométrico e dietético em nipo-brasileiros com e sem síndrome metabólica. Arquivos Brasileiros de Endocrinologia \& Metabologia, v.55, n.2, p.134-145, 2011.

DE BIASE, S.G. et al. Dieta vegetariana e níveis de colesterol e triglicérides [Vegetarian diet and cholesterol and triglycerides levels]. Arquivos Brasileiros de Cardiologia, v.88, n.1, p.35-9., 2007.

DUARTE, E.R. A mulher e o envelhecimento: alterações cardiovasculares na mulher geriátrica. Revista da Sociedade de Cardiologia do Rio Grande do Sul, v.12, p.1-6, 2007.

FETT, C.A. et al. Mudanças no estilo de vida e fatores de risco para doenças crônicas não transmissíveis e sistema imune de mulheres sedentárias. Revista de Nutrição, v. 22, n. 2, p. 245-255, 2009. Disponível em:

$<\mathrm{http} / / /$ dx.doi.org/10.1590/S1415-52732009000200007>.

Acesso em: $17 \mathrm{dez} .2017$.

FERREIRA, C.C.C.; PEIXOTO, M.R.G.; BARBOSA, M.A. Prevalência de fatoress de risco cardiovascular em idosos usuários do Sistema Único de Saúde de Goiânia. Arquivos Brasileiros de Cardiologia, São Paulo, v. 95, n. 5, p. 621628, 2010. Disponível em:

$<$ http://www.scielo.br/scielo.php?script=sci arttext\&pid=S0 066-782X2010001500010>. Acesso em: 17 dez. 2017.

FREITAS, E.V. et al. Importância da HDL para a ocorrência de doença cardiovascular no idoso. Arquivos Brasileiros de Cardiologia, v.93, n.3, p.231-238, 2009.

GRUNDY, S.M. et al. Implications of recent clinical trials for the National Cholesterol Education Program Adult Treatment Panel III Guidelines. Circulation, v.110, p. 22739, 2004 .

SÍNTESE de Indicadores Sociais. Uma Análise das Condições de Vida da População Brasileira 2010. Disponível em:

$<$ http://www.ibge.gov.br/home/estatistica/populacao/condic aodevida/indicadoresminimos/sinteseindicsociais2010/SIS 2010.pdf>. Acesso em: 24 mar. 2016.

LEMOS JÚNIOR, H.P., LEMOS, A.L.A. Gengibre. Revista Diagnóstico e Tratamento, v.15, n.4, p.174-178, 2010.

KRAUSE, M.P.; HALLAGE, T.; MICULIS, C.P. Análise do perfil lipídico de mulheres idosas de Curitiba - Paraná. Arquivos Brasileiros de Cardiologia, v. 90, n. 5, p.327$332,2008$.

KRIS-ETHERTON, P.M.; HARRIS, W.S.; APPEL, L.J. Fish consumption, fish oil, ômega-3 fatty acids, and cardiovascular disease. Circulation, v. 106, n. 21, p. $2747-$ 2757, 2002.

KRUG, R.R.; LOPES, M.A.; MAZO, G.Z. Barreiras e facilitadores para a prática de exercícios físicos de longevas inativas fisicamente. Revista Brasileira

de Medicina do Esporte, v. 21, n.1, p. 57-64, 2015.

MACKNESS, M.I.; DURRINGTON, P.N.; MACKNESS, B. How high-density lipoprotein protects against the effect of lipid peroxidation. Current Opinion in Lipidology, v. 11 , n. 4, p. $383-388,2000$

MCALISTER, F.A. et al. Randomised trials of secondary prevention programmes in coronary heart disease: systematic review. British Medical Journal, v. 323, n. 7319, p. 957-962, 2001.

MENDES, T.A.B.; WAKSMAN, R.D.; FARAH, O.G.D. Manual de Especialização - Geriatria e Gerontologia. 1. ed. Barueri: Manole, 2005.

NAGASHIMA, H.; KAZANUKI, H. The status of lipid management in 1836 patients with coronary artery disease: a multicenter survey to evaluate the percentage of japonese coronary artery disease patients achieving the target lowdensity lipoprotein cholesterol level specified by the Japan
Atherosclerosis Society. Journal of Atherosclerosis and Thrombosis, v.12, p.338-342, 2005.

OLIVEIRA, R.G.; PEDROSO, E.R.P. Blackbook - Clínica Médica. 2. ed. Belo Horizonte: Blackbook, 2014.

OLIVEIRA, T.C.; MDEIROS, W.R.; LIMA, K.C. Diferenciais de mortalidade por causas nas faixas etárias limítrofes de idosos. Revista Brasileira de Geriatria e Gerontologia, v.18, n.1, p. 85-94, 2015.

ORNISH, D. et al. Can lifestyle changes reverse coronary heart disease? The Lifestyle Heart Trial. The Lancet, v. 336 , n. 8708, p.129-33, 1990.

PIRICH, C.; BISOVSKY, S.; SINZINGER, H. Economic aspects of therapy for lipid metabolism disorders. Wiener klinische Wochenschrift, v. 104, n.14, p.401-408, 1992.

RADER, D.J. Regulation of reverse cholesterol transport and clinical implications. American Journal of Cardiology, v. 92, n. 4, p. 42-49, 2003.

ROMERO, T. Cardiac rehabilitation as a first step in the secondary prevention of coronary heart disease. Revista Medica De Chile, v.128, n. 8, p. 923-934, 2000.

RUTLEGE, J.C. et al. Lifestyle modification program in management of patients with coronary artery disease: the clinical experience in a tertiary care hospital. Journal of Cardiopulmonary Rehabilitation and Prevention, v.19, n.4, p.226-34, 1999.

SCHERR, C. et al. Intervenção nos hábitos de vida em uma instituição pública. Arquivos Brasileiros de Cardiologia. 2009. Disponível em: $<$

http://www.scielo.br/pdf/abc/v94n6/aop05110.pdf>. Acesso em: 5 mai. 2016.

SCHULER, G.; ADAMS, V.; GOTO, Y. Role of exercise in the prevention of cardiovascular disease: results, mechanisms, and new perspectives.

European Heart Journal, v. 62, n. 5, p. 634-637, 2013.

V DIRETRIZ Brasileira de Dislipidemias e Prevenção da Aterosclerose. Arquivos Brasileiros de Cardiologia, v. 101, n. 4, p. 20, 2013. Disponível em:

$<$ http://publicacoes.cardiol.br/consenso/2013/V Diretriz Br asileira_de_Dislipidemias.pdf $>$. Acesso em: 17 dez. $201 \overline{7}$.

TOLEDO, M.T.T. de; ABREU, M.N.; LOPES, A.C.S. Adesão a modos saudáveis de vida mediante aconselhamento por profissionais de saúde. Revista de Saúde Pública, v. 47, n. 3, p. 540-548, 2013. Disponível em: <http://dx.doi.org/10.1590/S0034-

8910.2013047003936>. Acesso em: 17 dez. 2017.

VACANTI, L.J. et al. A baixa taxa de obtenção da meta do LDL colesterol numa população de baixa renda. Arquivos Brasileiros de Cardiologia, v. 85. n. 3, p.162-165, 2005.

VERAS, R.P. Prevenção de doenças em idosos: os equívocos dos atuais modelos. Cadernos de Saúde Pública, v. 28, p.1834-1840, 2012.

WEST, R.O.; HAYES, O.B. Diet and serum cholesterol levels. A comparison between vegetarians and nonvegetarians in a Seventh-day Adventist Group. The American Journal of Clinical Nutrition, v. 21, n. 8, p. 853-62, 1968.

WILSON, P. et al. Prediction of coronary heart disease using risk factors (Framingham study). Circulation, v. 97, n. 18 , p. $1837-1847,1998$.

YUSUF, S. et al. Effect of potentially modifiable risk factors associated with myocardial infarction in 52 countries (the INTERHEART study): case-control study. The Lancet, v. 364, p. 937-952, 2004. 